\title{
NOTES
}

\section{Thiopeptide Non-producing Streptomyces Species Carry the tip $A$ Gene: A Clue to Its Function}

\author{
BONG-SIK YUN ${ }^{a, b}$, TOMOMI HIDAKA ${ }^{\mathrm{a}}$, \\ TOMOHISA KuZUYAma a and HARUO SETO ${ }^{\mathrm{a}, \dagger, *}$ \\ ${ }^{a}$ Institute of Molecular and Cellular Biosciences, \\ The University of Tokyo, \\ Bunkyo-ku, Tokyo 113-0032, Japan \\ ${ }^{\mathrm{b}}$ Korea Research Institute of Bioscience and Biotechnology, \\ P.O. Box 115, Yusong, Taejon 305-600, Korea
}

(Received for publication October 4, 2000)

Thiopeptide antibiotics are distinguished by structural features containing thiazole(s) and/or oxazole(s) with highly modified amino acids in mono-, di- or tri-cyclic peptide ring(s). Several thiopeptides are known to induce the biosynthesis of many proteins of unknown function in Streptomyces lividans ${ }^{1)}$. Two of these proteins, TipAL and TipAS, were characterized and their corresponding genes containing 'the promoter region (ptipA) were cloned and sequenced ${ }^{1}$. TipAL is a regulatory protein which autogenously activates transcription of its own promoter after forming an irreversible complex with thiostrepton or other related thiopeptide antibiotics ${ }^{2)}$, and this transcriptional activation of ptipA by TipAL results in the overexpression of TipAS, an in-frame translation product of the tipAL gene $^{2,3)}$. TipAS also forms a complex with thiopeptide compounds but does not activate transcription of its promoter ptipA. These proteins require the cyclic core and dehydroalanine side chains in the structures of thiopeptides for recognition and transcriptional activation ${ }^{3)}$. Although disruption of the tipA gene weakly increased antibiotic susceptibility in S. lividans, its functions are still under investigation.

The inducible promoter ptipA has been cloned into a series of vectors to allow regulated expression of genes in Streptomyces and those vectors have been employed to give a very sensitive and specific microbiological disc assay to screen for compounds inducing their transcription. Our screening to find tipA promoter-inducing compounds of microbial origin has resulted in the isolation of only the thiopeptide class of antibiotics ${ }^{4 \sim 8)}$. This finding led us to hypothesize that the tipA gene may be related to the production of thiopeptide antibiotic or the resistance specific against thiopeptides in Streptomyces. In this paper, we investigated the distribution of the tip $A$ gene in several different Streptomyces species to study the relationship between thiopeptide production and the tipA gene as a preliminary step to understand the function of the tipA gene.

Nine thiopeptide producing strains were selected as producers of tipA promoter-inducing principles, and nine thiopeptide nonproducing Streptomyces were randomly selected for this experiment (Table 1). Thiopeptide production by these strains was confirmed by resistance to thiostrepton, a representative thiopeptide antibiotic, and Southern blot analysis of the chromosomal DNAs isolated from these Streptomyces species with the thiostrepton resistant gene $(t s r)^{9)}$ as a probe. All the thiopeptide producing strains were resistant at concentrations up to 500 $\mu \mathrm{g} / \mathrm{ml}$ thiostrepton, while nonproducing strains were sensitive with different MIC values of 0.16 to $4 \mu \mathrm{g} / \mathrm{ml}$, as shown in Table 1. This result showed that all the thiopeptide producing strains possess the $t s r$ gene. This implication was supported by the Southern blot analysis using the $t s r$ gene. The cultures of Streptomyces species were grown at $30^{\circ} \mathrm{C}$ for one day in $10 \mathrm{ml}$ of GPY medium (glucose $1.0 \%$, polypepton $0.5 \%$, yeast extract $0.4 \%, \mathrm{MgSO}_{4} \cdot 7 \mathrm{H}_{2} \mathrm{O} 0.05 \%$, $\mathrm{K}_{2} \mathrm{HPO}_{4} \quad 0.1 \%$ ). The mycelia were washed with $25 \%$ sucrose and then disrupted by lysozyme. The chromosomal DNAs from each culture were obtained through the procedures of phenol-chloroform extraction and ethanol precipitation and were digested by $\mathrm{BamHI}$ for the Southern blot analysis. Two primers for the tsr gene corresponding to the nucleotides 391 402 (12-mer, CCCGCGGTGCAG) and 1117 1131 (15-mer, ATCGCGCTGCACGAG) were synthesized and used to prepare a $t s r$ probe by PCR. All thiopeptide producing strains hybridized with this DNA probe, while the nonproducing strains did not as shown in Fig. 1. This result was confirmed by repeated hybridization. We then investigated the distribution of the tipA gene

\footnotetext{
${ }^{\dagger}$ Present address: Faculty of Applied Bio-Science, Tokyo University of Agriculture, 1-1-1 Sakuragaoka, Setagaya-ku, Tokyo, Japan.

* Corresponding: haseto@nodai.ac.jp
} 
Table 1. Thiopeptide producing and nonproducing Streptomyces species used in this experiment.

\begin{tabular}{|c|c|c|c|c|c|c|}
\hline Strain & Produced compound & $\mathrm{MIC}(\mu \mathrm{g} / \mathrm{ml})^{a}$ & Lane No. ${ }^{b}$ & $T s r$ probing $^{c}$ & TipA probing ${ }^{c}$ & TipA $\mathrm{PCR}^{d}$ \\
\hline Streptomyces sp. DW76 & Promothiocins & $>500$ & 1 & + & - & nt \\
\hline Streptomyces sp. SF2741 & Promothiocins & $>500$ & 2 & + & - & nt \\
\hline S. morookaensis & Thioxamycin, thioactin & $>500$ & 3 & + & \pm & - \\
\hline S. rochei & Thiotipin & $>500$ & 4 & + & - & nt \\
\hline Streptomyces sp. CL10 & Thiotipin & $>500$ & 5 & + & - & nt \\
\hline Streptomyces sp. CR63 & Berninamycin & $>500$ & 6 & + & - & $\mathrm{nt}$ \\
\hline Streptomyces sp. DX49 & Promothiocins & $>500$ & 7 & + & - & $\mathrm{nt}$ \\
\hline Streptomyces sp. & Nosiheptide & $>500$ & 8 & + & - & $\mathrm{nt}$ \\
\hline S. azureus & Thiostrepton & $>500$ & 9 & + & - & nt \\
\hline S. fradiae & - & 0.16 & 10 & - & - & $\mathrm{nt}$ \\
\hline S. roseoflavus & - & 0.16 & 11 & - & + & + \\
\hline S. antibioticus IFO3126 & - & 0.16 & 12 & - & + & + \\
\hline S. aureofasciculus & - & 4 & 13 & - & + & \pm \\
\hline S. lavendulae & - & 0.8 & 14 & - & \pm & - \\
\hline S. citricolor & - & 0.16 & 15 & - & + & + \\
\hline S. moderatus & - & 4 & 16 & - & - & $\mathrm{nt}$ \\
\hline S. hygroscopicus & - & 4 & 17 & - & - & $\mathrm{nt}$ \\
\hline S. lividans & - & 0.8 & 18 & - & + & + \\
\hline Probe & & & 19 & & & \\
\hline
\end{tabular}

${ }^{a}$ Antimicrobial activity of thiostrepton against the thiopeptide producing and nonproducing strains. MIC indicates the minimum inhibitory activity. ${ }^{b} \mathrm{~L}$ ane numbers for Figs. 1-3. ${ }^{\circ}$ Southern blot analyses using thiostrepton resistance gene (tsr) and tipA gene probes, +: hybridized, -: not hybridized, \pm : not clear. "PCR amplification of the tipA gene, +: amplified, -: not amplified, \pm : amplified with a different size, nt: not tested.

among thiopeptide producing and nonproducing Streptomyces species by hybridization of their chromosomal DNAs with the tipA gene. The probes for hybridization (nucleotides $253 \sim 879$ ) were prepared by PCR using two synthetic primers [nucleotides 253 283 (31-mer, ACCTCGACCGGCTGCAGCAGATCCTGTTCTA) and 849 879 (31-mer, ATCCTCGCCAACGCCGTCCGGCACACCCCCT) $]^{1)}$. While thiopeptide producing strains did not show hybridization with the tipA probe at all, five of nine thiopeptide nonproducing strains (lanes 11, 12, 13, 15, and 18) showed positive results and lanes 3 and 14 were ambiguous, as shown in Fig. 2. This result was confirmed by repeated hybridization. Namely, the hybridized bands were found in 5 of 9 thiopeptide nonproducing strains examined.

Confirmation of these bands was carried out by PCR analysis (Fig. 3). The chromosomal DNAs of the strains $S$. morookaensis (lane 3), S. roseoflavus (lane 11), $S$. antibioticus (lane 12), S. aureofasciculus (lane 13), S. lavendulae (lane 14), S. citricolor (lane 15), and S. lividans (lane 18) were amplified by PCR with the primer sets indicated above. $S$. roseoflavus, $S$. antibioticus, and $S$. citricolor yielded PCR products of the same size as that of
S. lividans (lane 18) while $S$ morookaensis, a thiopeptide producing strain, produced some nonspecific bands and $S$. aureofasciculus produced a PCR product with a different size, and $S$. lavendulae did not produce any product. These results imply that at least four species, $S$. roseoflavus, $S$. antibioticus, S. citricolor, and S. lividans have the tipA gene in their chromosomal DNA. Thus the tipA gene was found in 4 , possibly 5 , of the 6 thiopeptide nonproducing Streptomyces species examined.

The tipA gene does not seem to be directly involved in thiopeptide production, because the gene exists only in some thiopeptide nonproducing species of Streptomyces. It was reported that the disruption of the tipA gene in $S$. lividans increased sensitivity to thiopeptide antibiotics up to about 5 times $^{3)}$. Thus, the tip $A$ gene is suggested as serving as an autogeneously controlled resistance system specific to thiopeptides. We notice that the tipA gene does not exist in thiopeptide producing strains with the $t s r$ gene, high-level thiopeptide resistance system, and the expression of tipA gene is dependent on the intracellular level of free thiopeptide compounds. In response to thiopeptides, TipAL activates transcription of its own promoter and thus results in the expression of TipAS, an alternate in-frame translation 
Fig. 1. Southern blot analysis of Streptomyces species DNA using thiostrepton resistance gene $(t s r)$ probe.

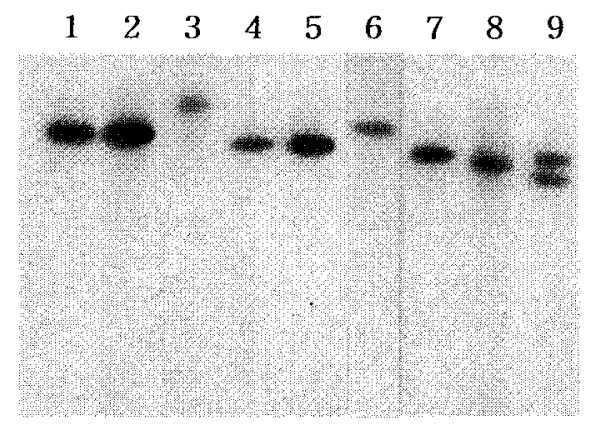

$\begin{array}{llllllllll}10 & 11 & 12 & 13 & 14 & 15 & 16 & 17 & 18 & 19\end{array}$

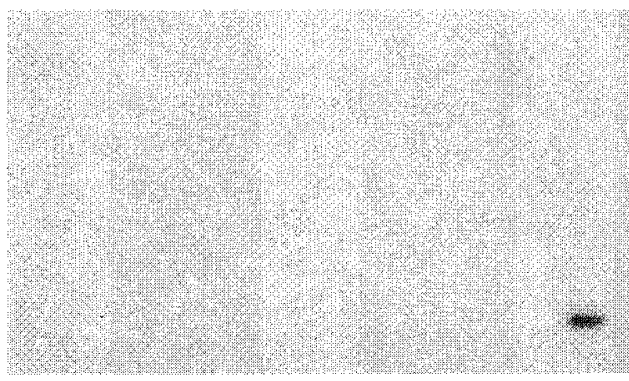

BamHI-digested DNA from the thiopeptide producing and nonproducing Streptomyces species listed in Table 1 was analyzed by a Southern blot probed with the $t s r$ probe. Lanes $1 \sim 9$ contain DNA from thiopeptide producing Streptomyces strains and lanes $10 \sim 18$ contain DNA from thiopeptide nonproducing strains. Lane 19 is the hybridization probe (nucleotides 391 1131) of the tsr gene.

Fig. 3. PCR amplification of the tipA gene using DNA from various Streptomyces species.

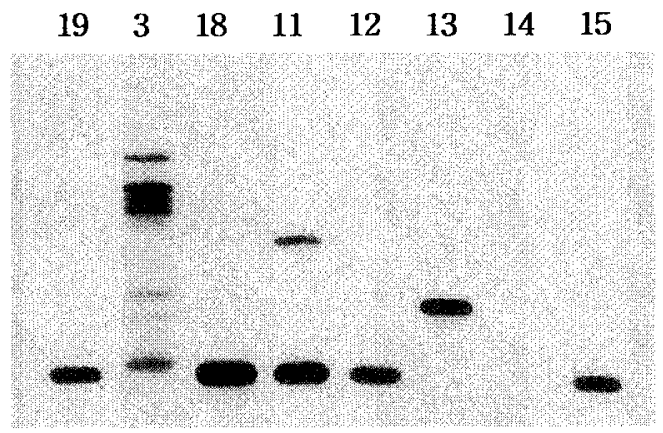

PCR amplification was carried out using two synthetic primers [nucleotides 253 283 (31-mer, ACCTCGACCGGCTGCAGCAGATCCTGTTCTA) and $849 \sim 879$ (31-mer, ATCCTCGCCAACGCCGTCCGGCACACCCCCT)] under the same conditions. DNAs used for PCR amplification are as described in Table 1.
Fig. 2. Southern blot analysis of Streptomyces species DNA using the tipA gene probe.
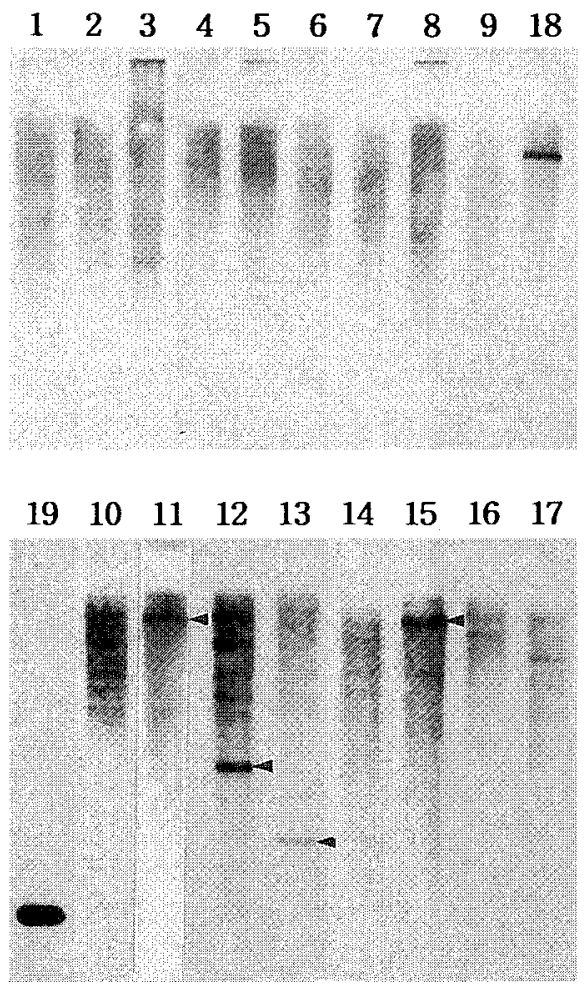

BamHI-digested DNA from the thiopeptide producing and nonproducing Streptomyces species listed in Table 1 was analyzed by a Southern blot probed with the tipA gene. Lanes $1 \sim 9$ contain DNA from thiopeptide producing Streptomyces strains and lanes $10 \sim 17$ contain DNA from thiopeptide nonproducing strains. Lane 18 contains DNA from S. lividans which is used as a positive control and lane 19 is the hybridization probe (nucleotides $253 \sim 879$ ) of the tipA gene.

product of the same gene. The expression of TipAS may control activation of the TipAL-dependent promoter by decreasing the intracellular concentration of free thiopeptides. The modulation of intracellular free thiopeptide level by the binding of thiopeptides with tipA gene products (TipAL and TipAS, antibiotic-inactivating proteins) would provide a self-contained antibiotic resistance system to diminish inhibition by transient exposure to thiopeptides. However, since $S$. moderatus and $S$. hygroscopicus without the tipA gene showed higher resistance to thiostrepton than those of $S$. roseoflavus, $S$. antibioticus, $S$. citricolor and $S$. lividans with the tipA gene, as shown in Table 1, this mechanism seems to be extremely low-level resistance or transient stress response system for 
Streptomyces very sensitive to thiopeptide compounds. Thus, thiopeptide producing Streptomyces with high-level antibiotic resistance system would not require the tip $A$ gene, a low-level thiopeptide resistance system.

Through this preliminary study on the function of tipA gene, we found that the tipA gene often existed in thiopeptide nonproducing Streptomyces but not in thiopeptide producing strains. Although one of major roles of the tipA gene is suggested to be antibiotic resistance specific to thiopeptides, we can not eliminate other possible physiological functions. It also should be investigated whether S. roseoflavus, $S$. antibioticus, and $S$. citricolor as $S$. lividans will be more sensitive strains to thiopeptides when the tipA gene is knocked out. Other roles of the tipA gene remain to be investigated.

\section{References}

1) Murakami, T.; T. G. Holt \& C. J. Thompson: Thiostrepton-induced gene expression in Streptomyces lividans. J. Bacteriol. 171: 1459 1466, 1989

2) Holmes, D. J.; J. L. CAso \& C. J. Thompson: Autogenous transcriptional regulation of a thiostreptoninduced gene in Streptomyces lividans. EMBO J. 8: 3183 3191, 1993

3) Chiu, M. L.; M. Folcher, T. Katoh, A. M. Puglia, J. Vohradsky, B.-S. Yun, H. Seto \& C. J. Thompson:
Broad spectrum thiopeptide recognition specificity of the Streptomyces lividans TipAL protein and its role in regulating gene expression. J. Biol. Chem. 274: 20578 20586, 1999

4) Yun, B.-S.; T. Hidaka, K. Furihata \& H. Seto: Promothiocins $\mathrm{A}$ and $\mathrm{B}$, novel thiopeptides with a tipA promoter inducing activity produced by Streptomyces $\mathrm{sp}$. SF2741. J. Antibiotics 47: 510 514, 1994

5) Yun, B.-S.; T. Hidaka, K. Furuhata \& H. Seto: Microbial metabolites with a tipA promoter inducing activity. II. Geninthiocin, a novel thiopeptide produced by Streptomyces sp. DD84. J. Antibiotics 47: 969 975, 1994

6) Yun, B.-S.; T. Hidaka, K. Furihata \& H. Seto: Thiotipin, a novel thiopeptide with a tipA promoter inducing activity produced by Streptomyces sp. DP31. Tetrahedron 50: 11659 11664, 1994

7) Yun, B.-S.; T. Hidaka, K. Furihara \& H. Seto: Microbial metabolites with a tipA promoter inducing activity. III. Thioxamycin and its novel derivative, thioactin, two thiopeptides produced by Streptomyces $\mathrm{sp}$. DP94. J. Antibiotics 47: 1541 1545, 1994

8) Yun, B.-S. \& H. SETO: Promoinducin, a novel thiopeptide produced by Streptomyces sp. SF2741. Biosci. Biotech. Biochem. 59: 876 880, 1995

9) BibB, M. J.; M. J. BiBB, J. M. WARD \& S. N. CoheN: Nucleotide sequences encoding and promoting expression of three antibiotic resistance genes indigenous to Streptomyces. Mol. Gen. Genet. 199: $26 \sim 36,1985$ 